\title{
A feasibility study of the dosimetric compliance criteria of the NRG-HN002 head and neck clinical trial across different radiotherapy treatment planning systems and delivery techniques:A model for optimizing initial trial launch
}

\begin{abstract}
The aim of this study was to assess the feasibility of the dosimetric compliance criteria of the NRG-HNO02 clinical trial, using different treatment planning systems and different delivery techniques, with the aim of optimizing the initial launch of this national head and neck cancer radiotherapy trial. Five benchmark patients meeting the eligibility criteria for NRG-HN002 [No Icon Annotation] were included in this study. Contours for target volumes and organ at risks were drawn according to the study guidelines. Two IMRT and two VMAT plans were developed for each patient using Varian Eclipse and the auto-planning module in Philips Pinnacle. Dose volume histograms of the plans were analyzed and compared using MIM software and tested against the compliance criteria of the protocol. All dosimetric compliance criteria were met using the two treatment planning systems and delivery techniques, except the maximum dose for PTV_4800 when it overlapped with higher dose PTVs. The coverage for the three PTVs (PTV 6000, PTV 5400 and PTV 4800) was achieved and also the PTV maximum doses were not exceeded in both IMRT and VMAT plans. The average maximum dose in all plans for SpinalCord 05, Spinal Cord

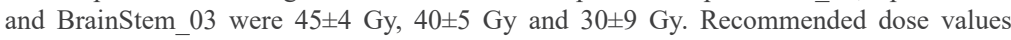
for other OARs (Parotid Contra, larynx, pharynx, Sub Mandibular Contra, Oral Cavity, Esophagus_Up, Mandible and NonPTV) were also achieved with minor deviations in very few occasions. The maximum dose of PTV 4800 criterion was removed from the protocol based on the results of this study. For radiotherapy-based clinical trials, this feasibility testing process is a model for reducing planning deviations and the necessity for amendments to the dosimetric criteria subsequent to activation.
\end{abstract}

Keywords: NRG-HN002, dosimetric criteria, clinical trial, IMRT, VMAT
Volume 5 Issue 5 - 2016

\author{
Tawfik Giaddui,' Jialu Yu,' Anthony Doemer,' \\ Martha Matuszak, ${ }^{3}$ Francisco Nunez, ${ }^{4}$ Karen \\ Vineberg, ${ }^{3}$ Ping Xia, ${ }^{5}$ Sue S Yom, ${ }^{6}$ Ying $\mathrm{Xiao}^{7}$ \\ 'Thomas Jefferson University, USA \\ ${ }^{2}$ Henry Ford Hospital, USA \\ 'University of Michigan, USA \\ ${ }^{4}$ Philips Radiation Oncology Systems, USA \\ ${ }^{5}$ Cleveland Clinic, USA \\ 'University of California, USA \\ University of Pennsylvania, USA
}

\section{Correspondence: Tawfik Giaddui, Sidney Kimmel Cancer} Center, Thomas Jefferson University, USA, Tel 215955 8855, Fax 215955 04I2, Email tawfik.g.giaddui@jefferson.edu

Received: August 25, 2016 | Published: September 20, 2016

\section{Introduction}

NRG HNO02 ${ }^{1}$ is a randomized phase II trial for patients with good- risk human papilloma virus (HPV)- associated oropharyngeal cancer (OPC). The trial will evaluate two new treatment options, one consisting of chemo radiotherapy and one of radiotherapy alone. The prescription dose is $60 \mathrm{~Gy}$ (2.0 Gy/fraction) over six weeks for arm 1 and over five weeks for arm 2 . All patients will be managed with intensity modulated radiation therapy (IMRT) delivered with mega voltage photons. The goal of the trial is to evaluate whether these two modestly less-intensive therapies result in the same excellent outcomes and low treatment-associated toxicity observed in the RTOG $012^{2}$ and RTOG $0522^{3}$ trials of oropharyngeal cancer. Further details on the NRG HN002 clinical trial can be found elsewhere.

The compliance criteria of clinical trials are often used as constraints for treatment planning and are always used for plan scoring upon final review. ${ }^{4}$ Upon review at the Imaging and Radiation Oncology Core (IROC) RT QA center, treatment plans of patients enrolled in NRGOncology clinical trials are given scores of 1,2 or 3. A treatment plan is given a score of 1 if it exactly meets the criteria; given a score of 2 if it falls within the variation acceptable range, and given a score of 3 and rejected if it violates the variation acceptable range. The method used for designing compliance criteria should optimally examine a large number of treatment plans that are considered to be the result of significant effort on the part of experienced treatment planners. ${ }^{4}$ Such a process should take place prior to the activation of the clinical trial. However, in many cases, new clinical trials uses dosimetric criteria and constraints of similar and previous trials and this may lead to repeated amendments of the newly developed trials when many participating institutions cannot meet the dosimetric constraints. Such repeated amendments are undesirable and may lead to confusion, especially in multi-institutional clinical trials. RTOG 1005, for example suffered from high failure rates in rapid review cases, ${ }^{5}$ as $35 \%$ of the rapid review plans failed to meet the compliance criteria of the protocol (either contouring or dosimetric criteria). Among these plans, $12 \%$ of the plans failed to meet the dosimetric criteria and the protocol was amended to relax the dosimetric criteria for the contralateral breast. NRG-Oncology, as a part of the quality assurance of its clinical trials, started to pro-actively test the dosimetric compliance criteria of any clinical trial and establish its feasibility prior to activation. As such, unnecessary future amendments, which may lead to confusion and also high failure rates during the RTQA process, can be easily avoided. Such a step represents an important improvement in the launch of a new clinical trial. In a previous study, the dosimetric compliance criteria of the RTOG 1308 clinical trial was investigated during the design stage of the trial. ${ }^{4}$ The investigation study analyzed 26 pairs 
(each pair consisted of IMRT and proton passive scattered therapy (PSPT) plan) of treatment plans submitted by two major institutions. The plans were developed using institutional protocols that have similar guidelines to RTOG 1308 and the doses were scaled to the prescription dose of RTOG 1308. The study ${ }^{4}$ helped in establishing feasible dosimetric criteria for RTOG 1308. This paper reports on assessing the dosimetric compliance criteria of clinical trials (applied to NRG HN002 trial) using a different approach. Here, DICOM CT and structures data of five bench mark cases were sent to different academic institutions and different treatment planning systems (Eclipse and Pinnacle) and delivery techniques (IMRT and VMAT) were used as a part of the trial planning process.

\section{Methods and materials}

\section{Dosimetric compliance criteria of NRG-HN002}

Tables $1 \& 2$ lists the required dosimetric compliance criteria and the recommended dose acceptance criteria for some normal structures, respectively. The recommended dose criteria are used for planning guidance but not for plan evaluation.

\section{Treatment planning instructions and priorities}

Table 3 summarizes the treatment planning instructions and priorities for target volumes and organs at risks.

Table I Dosimetric compliance criteria of NRG HN002 clinical Trial

\begin{tabular}{|c|c|c|c|}
\hline Name of structure & Dosimetric parameters & Per protocol dose (Gy) & Variation acceptable \\
\hline & D95\%*(Gy) & 60 & $>60$ and $\leq 63$ \\
\hline \multirow[t]{2}{*}{ PTV_6000 } & D99\%(Gy) & $\geq 55.8$ & $\geq 54$ \\
\hline & $\operatorname{Dmax}^{* *}(\mathrm{~Gy})$ & $\leq 66$ & $\leq 69$ \\
\hline CTV_6000 & V60 Gy (\%) & $\geq 99 \%$ & 95 to $99 \%$ \\
\hline \multirow{2}{*}{ PTV_5400 } & D95\%*(Gy) & $\geq 54$ & $\geq 51.3$ \\
\hline & D99\%(Gy) & $\geq 50.2$ & $\geq 48$ \\
\hline \multirow[t]{2}{*}{ CTV_5400 } & V54 Gy (\%) & $\geq 99 \%$ & 95 to $99 \%$ \\
\hline & D95\%*(Gy) & $\geq 48$ & $\geq 44$ \\
\hline \multirow[t]{2}{*}{ PTV_4800 } & D99\%(Gy) & $\geq 44.6$ & $\geq 43.2$ \\
\hline & Dmax**(Gy) & $\leq 55.2$ & $\leq 57.6$ \\
\hline CTV_4800 & V48 Gy (\%) & $99 \%$ & 95 to $99 \%$ \\
\hline Spinal Cord_05 & $\operatorname{Dmax} * *(G y)$ & $\leq 48$ & $\leq 50$ \\
\hline Spinal Cord & $\operatorname{Dmax} * *(G y)$ & $\leq 45$ & $\leq 48$ \\
\hline Brain Stem_03 & $\operatorname{Dmax} * *(G y)$ & $\leq 50$ & $\leq 52$ \\
\hline
\end{tabular}

*D95 \%( Gy) $=$ dose to $95 \%$ of volume.

**Dmax $=$ maximum dose to $0.03 \mathrm{cc}$ of the volume.

Table 2 Recommended dose acceptance criteria for other normal tissue, but not to be used for plan score

\begin{tabular}{ll}
\hline Structure & Recommended dose acceptance criteria \\
\hline Parotid & Mean dose to one parotid $\leq 26$ Gy \\
Larynx & Mean dose $\leq 35 \mathrm{~Gy}$ \\
Pharynx & Mean dose $\leq 40 \mathrm{~Gy}$ \\
Submandibula_R or Submandibula_L (ccontrlateral) & Mean Dose $\leq 39 \mathrm{~Gy}$ \\
Oral Cavity (Excludin PTV's) & Mean dose $\leq 32 \mathrm{~Gy}$ \\
Esophagus_Up & Mean dose $\leq 30 \mathrm{~Gy}$ \\
NonPTV & DIcc $<63 \mathrm{~Gy}$ \\
Mandible & D0.03cc $<63 \mathrm{~Gy}$ \\
\hline
\end{tabular}

Table 3 Treatment planning instructions and priorities

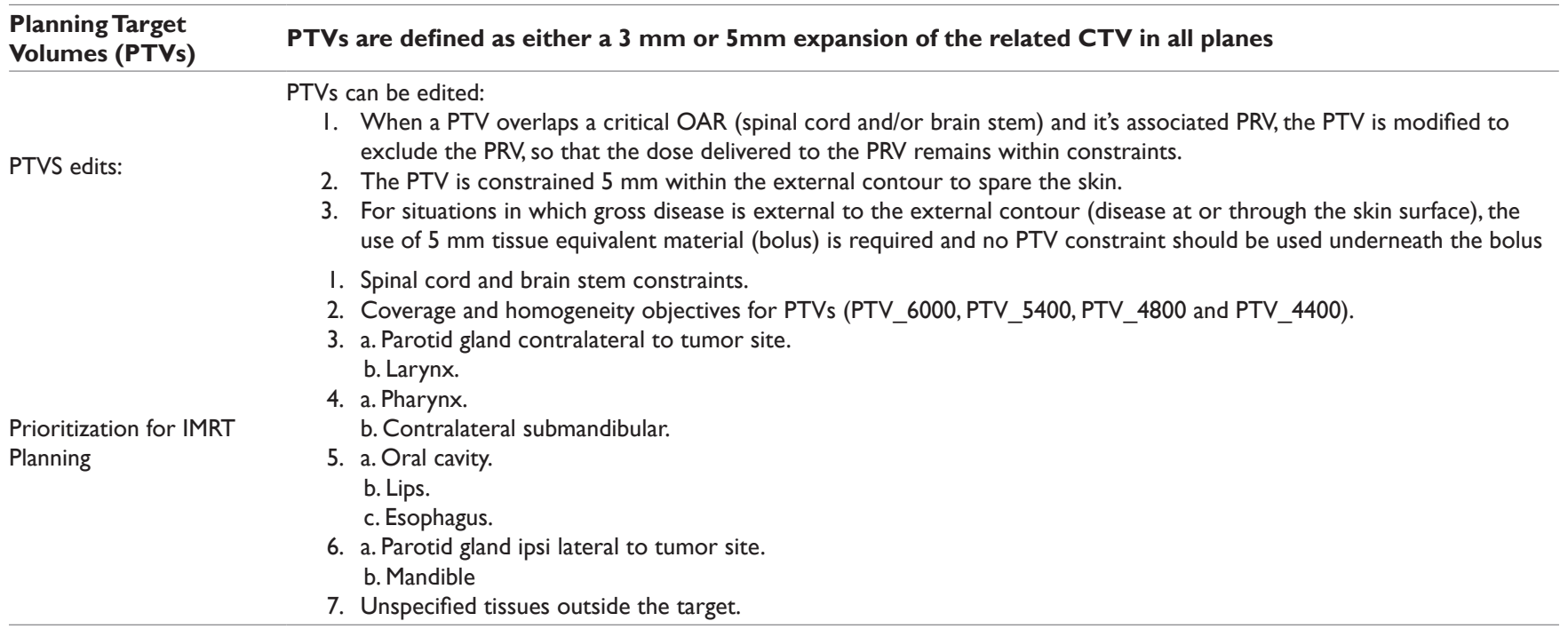


Table 4 Values of dosimetric parameters of the target volumes that are included in the dosimetric compliance criteria of NRG HN002 clinical trial

\begin{tabular}{|c|c|c|c|c|c|}
\hline \multirow{3}{*}{ Structure } & \multirow{3}{*}{$\begin{array}{l}\text { Dosimetric } \\
\text { parameter }\end{array}$} & \multicolumn{2}{|l|}{ VMAT } & \multicolumn{2}{|l|}{ IMRT } \\
\hline & & Varian eclipse & Philips pinnacle & Varian eclipse & Philips pinnacle \\
\hline & & Mean士STD & Mean $\pm S T D$ & Mean $\pm S T D$ & Mean士STD \\
\hline \multirow{3}{*}{ PTV_6000 } & D95\%(Gy) & $60.02 \pm 0.08$ & $60.05 \pm 0.04$ & $60.0 \pm 0.00$ & $60.03 \pm 0.03$ \\
\hline & D99\%(Gy) & $58.43 \pm 0.49$ & $59.30 \pm 0.10$ & $57.00 \pm 3.00$ & $59.18 \pm 0.11$ \\
\hline & $\operatorname{Dmax}(\mathrm{Gy})$ & $65.64 \pm 0.18$ & $64.17 \pm 0.53$ & $66.00 \pm 1.00$ & $64.48 \pm 0.62$ \\
\hline CTV_6000 & V 60Gy (\%) & $99.46 \pm \pm 0.44$ & $99.98 \pm 0.05$ & $98.00 \pm 2.00$ & $99.80 \pm 0.23$ \\
\hline \multirow{2}{*}{ PTV_5400 } & D95 \% (Gy) & $54.05 \pm 0.17$ & $54.91 \pm 0.49$ & $55.00 \pm 1.00$ & $54.99 \pm 0.56$ \\
\hline & D99 \% (Gy) & $52.77 \pm 0.72$ & $54.08 \pm 0.45$ & $53.00 \pm 2.00$ & $54.05 \pm 0.50$ \\
\hline \multirow[t]{2}{*}{ CTV_5400 } & V54 Gy (\%) & $99.84 \pm 0.15$ & $99.9 \pm 0.12$ & $99.70 \pm 0.42$ & $99.94 \pm 0.09$ \\
\hline & D95\% (Gy) & $48.18 \pm 0.79$ & $48.52 \pm 0.59$ & $49.44 \pm 0.94$ & $48.79 \pm 0.22$ \\
\hline \multirow[t]{2}{*}{ PTV_4800 } & D99\% (Gy) & $47.72 \pm 0.67$ & $48.24 \pm 0.12$ & $47.00 \pm 1.00$ & $47.98 \pm 0.42$ \\
\hline & Dmax (Gy) & $57.00 \pm 3.00$ & $57.00 \pm 6.00$ & $58.00 \pm 3.00$ & $62.00 \pm 2.00$ \\
\hline CTV_4800 & V 48 Gy (\%) & $99.67 \pm 0.31$ & $99.94 \pm 0.06$ & $99.87 \pm 0.11$ & $99.86 \pm 0.21$ \\
\hline
\end{tabular}

\section{Patient population and target definitions}

Five bench mark patients, who were treated on a previous clinical trial of HPV-associated oropharyngeal cancer patients, RTOG 1016 and who met the more selective inclusion criteria for NRG HN002, were identified for this study. There was diversity among the cases with respect to the primary site within the oropharynx (tonsil or base of tongue), primary tumor stage ( $\mathrm{T}$ stage), and nodal stage ( $\mathrm{N}$ stage). The contours of the target volumes and organ at risk were redrawn according to the guidelines of NRG HN002. Figure 1 gives examples of axial views of two CT images included in this study and showing planning target volume (PVT) and organs at risk (OARs).

\section{Treatment planning systems and delivery techniques}

Two treatment planning systems were used to plan the five cases included in this study. These systems were Varian Eclipse version 11.0.30 (Varian Medical Systems, Palo Alto, CA) and Philips Pinnacle version 9.10 (Philips Health Care, Fitchburg, WI). Plans were developed using either Intensity Modulated Radiation Therapy (IMRT) with nine beams at 40 degrees of separation from the anteroposterior axis and Volumetric Modulated Arc Therapy (VMAT) with two full arcs.

Plans in Pinnacle were developed using Pinnacle Auto-Planning. Pinnacle Auto-Planning is a software module available starting in version 9.10 that simplifies the planning process through the use of templates and automatic optimization tuning methods. It automatically mimics actions taken by an experienced dosimetrist to create an IMRT or VMAT plan. These actions include, but are not limited to, creating rings, managing structure overlaps between PTVs and OARs and controlling dose in hot and cold spots. The prioritized clinical goals defined in the template are translated into optimization objectives. The software is designed to continually optimize sparing of OARs, even beyond the clinical goals which were input by the planner, if possible, all while maintaining the user-specified dose uniformity in the PTV. Extra control regions of interest (ROIs) and objectives are added if necessary to automatically control dose uniformity to the target(s) and enforce the high priority goals of maximum dose. ${ }^{6}$

\section{Data analysis}

Dose volume histograms (DVHs) for all plans were compared using MIM Software, Inc., ${ }^{7}$ which was only used to display, compare and analyze DVHs. Dose parameters from the two types of plans (IMRT and VMAT) were compared using a paired t-test. Differences were considered significant if $\mathrm{p}<0.05$ ( 2 -sided).
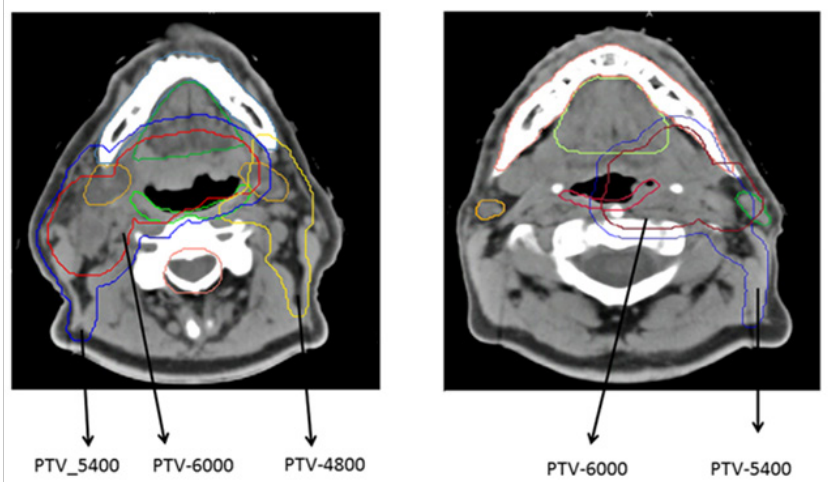

Figure I Examples of axial views of two CT images included in this study, showing PTVs and OARs.
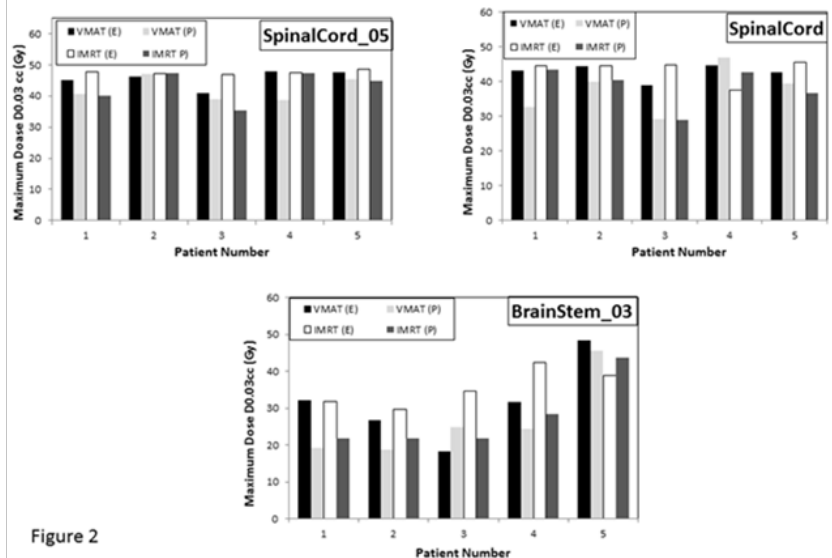

Figure 2 Histograms of maximum dose of spinal cord_05, spinal cord and brain stem 03 for all patients.

E, eclipse TPS; P, pinnacle TPS.

\section{Results}

\section{Compliance of IMRT and VMAT plans with NRG- HN002 dosimetric compliance criteria}

Table 4 lists the values for the dosimetric parameters of the target volumes that were obtained using the two treatment planning systems 
and the two delivery techniques. As can be seen, all dosimetric compliance criteria of the target volumes were met using the two planning techniques, with the exception of the PTV_4800 maximum dose criterion. Coverage requirements (D95\% (Gy) and D99\% (Gy) for the three PTVs (PTV_6000, PTV_5400 and PTV_4800) were met in all cases. The V60 Gy (\%) for CTV 6000, V54 Gy (\%) for CTV_5400 and V48 Gy (\%) for CTV_4800 were also met. The deviation of the obtained values for PTV 4800 maximum dose over the value defining an acceptable variation (maximum dose of 57.6 Gy within PTV_4800) ranged from $0.4 \%$ to $6.32 \%$ in VMAT plans and from $0.5 \%$ to $5.9 \%$ in IMRT plans. The unacceptable deviation rates (percentage of plans that failed to meet minimum criteria) with respect to this criterion were $40 \%$ and $60 \%$ amongst the VMAT and IMRT plans, respectively. Figure 2 shows histograms of the maximum doses (D0.03 cc) of Spinal Cord, SpinalCord_05 and BraiStem_03 for all patients. These structures are the only critical structures that are included in the dosimetric compliance criteria. As can be seen, these criteria are met for all patients included in the study. The average maximum dose in all plans for SpinalCord 05, Spinal Cord and BrainStem 03 were $45 \pm 4$ Gy, $40 \pm 5$ Gy and $30 \pm 9$ Gy. Figure 3 shows dose histograms for the contralateral parotid, larynx, pharynx and oral cavity and Figure 4 shows dose histograms of esophagus, mandible and Non PTV for all patients. These dosimetric paramenets (Figures $3 \& 4$ ) are used as guidance for planning and are not scored upon final review of the treatment plans. As can be seen, all recommended dose values were also achieved with some deviations in very few occasions. Both treatment planning systems and treatment techniques gave comparable estimates of the dose levels of critical structures. However, differences were observed occasionally.
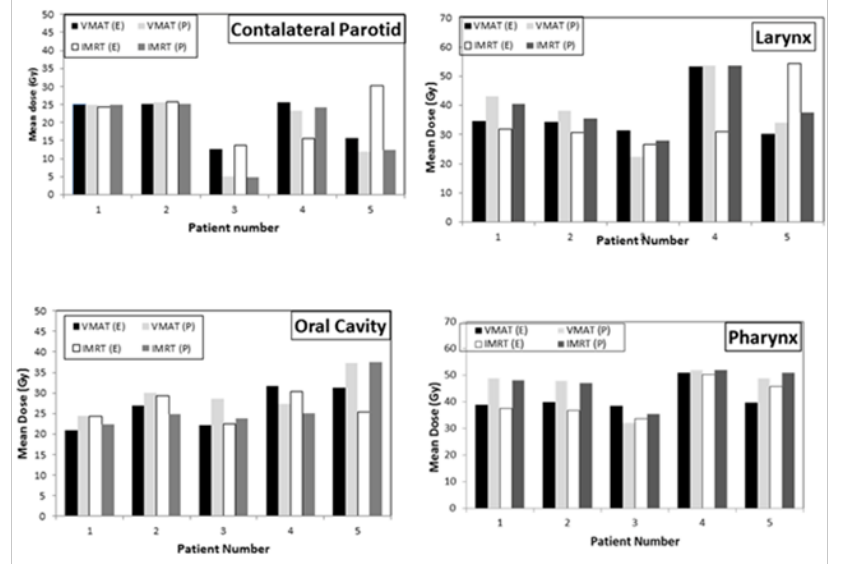

Figure 3 Dose histograms for the contralateral Parotid, larynx, pharynx and oral cavity for all patients.

E, eclipse TPS; P, pinnacle TPS.

\section{Discussion}

The current study was conducted during the development of the NRG HNO02 trial to assess the feasibility of the dosimetric compliance criteria of this clinical trial. Two major treatment techniques (IMRT and VMAT) and treatment planning systems were employed on a limited number of test patients deemed to be representative of those expected to enroll on the trial. The results of this study indicated that all dosimetric compliance criteria could be met with the exception of the maximum dose of PTV_4800 when it overlapped with PTV_6000. An unacceptable deviation rate of less than $10 \%$ may be tolerated but a higher rate indicates a consistent problem; therefore, the
PTV_4800 maximum dose criterion was removed from the protocol. The major objective of investigating whether the dosimetric criteria of this clinical trial were achievable, in advance of its activation, was successful. No further dosimetric amendments have been required to the protocol since activation.
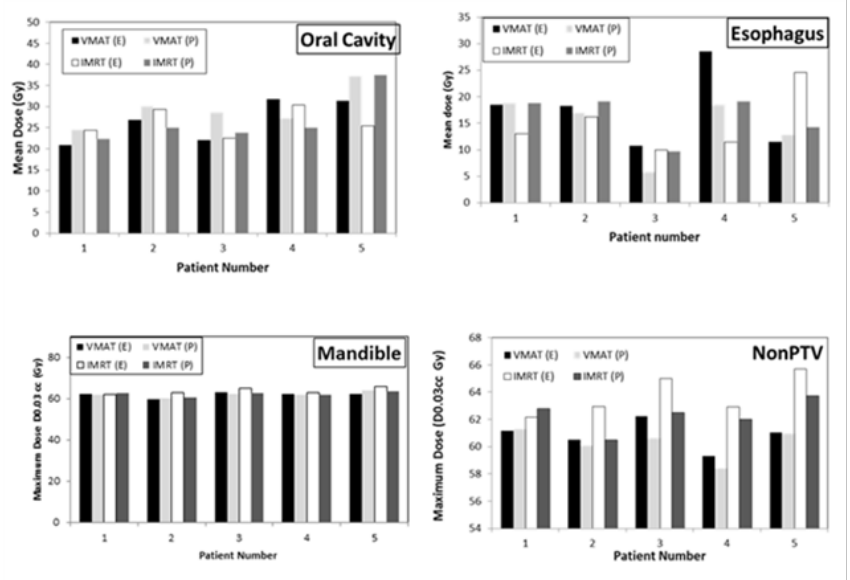

Figure 4 Dose histograms of esophagus, mandible and NonPTV for all patients. E, eclipse TPS; P, pinnacle TPS.

As our major goal during this process was to establish feasibility of the radiotherapy quality measures to be used in NRG HN002, we did not aim to conduct a formal comparison of VMAT with IMRT or Pinnacle with Eclipse plans. These two treatment delivery techniques and these two TPS were selected because they were deemed to be the most likely to be used by the various institutions participating in this national clinical trial. However, there were some variations in the results among the two delivery techniques and two TPS. In general, these variations were not statistically significant.

Other comparative treatment planning studies have yielded a similar conclusion of limited differences in results among systems. For instance, Holt et al. ${ }^{8}$ compared IMRT and VMAT plans generated by five institutions for five oropharyngeal patients using Pinnacle or Oncentra Master Plan. They used a prescription dose of 56 Gy to the elective PTV and Simultaneous Integrated Boost (SIB) to achieve a prescription PTV dose of 69.12 Gy. They reported a spinal cord maximum dose of 45.1 and 46.6 for VMAT and IMRT, respectively (compared to $40.1 \pm 5 \mathrm{~Gy}$, and $40.9 \mathrm{~Gy}$ in our study). They also reported, for VMAT and IMRT, respectively: a contralateral parotid mean dose of 22 Gy and 23 Gy (19.5 Gy and 20.1 Gy in our study); a submandibular mean dose of $53 \mathrm{~Gy}$ and $54.2 \mathrm{~Gy}$ (31.3 Gy and $37.3 \mathrm{~Gy}$ in our study); an oral cavity mean dose of 36.7 Gy and 39.4 Gy (28.1 and 26.6 Gy, in our study); and a larynx mean dose of 45.5 Gyand 46.5 Gy (37.5 Gy and 37.0 Gy in our study). It should be noted that the prescription dose for our plans was $60 \mathrm{~Gy}$ and in the plans by Holt et al. the prescription dose was $69.12 \mathrm{~Gy}$.

Clemente et al. ${ }^{9}$ compared IMRT, VMAT and helical tomotherapy for oropharyngeal cancer using SIB. They used three prescription dose levels in their study: 70; 63 and 58.1 Gy to regions of macroscopic, microscopic high risk and microscopic low risk. They reported a spinal cord D0.1ce dose of 35.8 Gy and 35.9 Gy for VMAT and IMRT, respectively (compared with a spinal cord maximum dose to D.03cc of 40.1 Gy and 40.9 Gy in our study). They also reported a D0.1cc dose for the spinal cord PRV (Cord $+4 \mathrm{~mm}$ ) of 39.4 Gy for both VMAT and IMRT (compared to a maximum dose to D.03 cc for spinal cord $+5 \mathrm{~mm}$ of 43.9 Gy and 40.9 Gy in our study). 
Dai et al. ${ }^{10}$ compared VMAT and IMRT plans for oropharyngeal carcinoma; their study included fifteen patients and they used two dose level prescription for PTVs (70 Gy and 56 Gy). For VMAT, they used both single arc ( $\operatorname{arc} 1)$ and double arc (arc2 plans). They reported a D0.1cc of the spinal cord of $41.1 \mathrm{~Gy}, 44.3 \mathrm{~Gy}$ and $40.0 \mathrm{~Gy}$ for IMRT, arc1 VMAT and arc2 VMAT plans (D.03cc was 40.9 Gy and 40.1 Gy for IMRT and arc2 VMAT in our study). In summary, taking into account slight differences in the evaluation criteria, our results are consistent within the reported values from other comparative planning studies and similar to these other studies; affirm the reasonableness of feasibility testing for multiple dose criteria across different systems.

\section{Conclusion}

All dosimetric compliance criteria for the newly developed head and neck NRG HN002 clinical trial were readily achieved using different treatment planning systems and techniques, with the exception of the PTV_4800 maximum dose. As a result of this study, the PTV 4800 maximum dose limit was removed from the dosimetric compliance criteria of the protocol. Both treatment planning systems (Eclipse and Pinnacle) gave similar plans in general. VMAT and IMRT plans using both Eclipse and Pinnacle TPS gave comparable results for the various dose parameters (within the uncertainty levels) of the OARs. This feasibility study, conducted in advance of trial activation, represents a practical improvement in trial planning processes. By establishing in advance that specified dosimetric criteria are feasible across a variety of common treatment planning techniques, the efficiency of a clinical trial launch and the consistency of collected data may be enhanced. In addition, based on the feasibility study, technical guidance can be provided to centers prior to trial launch.

\section{Acknowledgments}

This project was supported by grants U10CA180868, U10CA180822 and U24CA180803 and U24 RFA-CA-12-014 from the National Cancer Institute (NCI) and the Cure Grant from the Department of Health in Pennsylvania.

\section{Conflicts of interest}

The author declares no conflicts of interest.

\section{Funding}

None.

\section{References}

1. Sue Yom S, Jimmy Caudell J, John Waldron, et al. NRG-HN002: A Randomized Phase Ii Trial For Patients With p16 Positive, NonSmoking Associated, Loco regionally Advanced Oropharyngeal Cancer. Nrg Oncol Clin. 2014. p. 1-4.

2. Wheeler RH. Radiation Therapy Oncology Group Rtog 0129 A Phase Iii Trial Of Concurrent Radiation And Chemotherapy (Followed By Surgery For Residual Primary / N2-3 Nodal Disease) For Advanced Head And Neck Carcinomas. Radiation Therapy Oncology Group, USA. 2010

3. Axelrod R, Ang K, Rosenthal M. Rtog 0522 A Randomized Phase Iii Trial of Concurrent Accelerated Radiation and Cisplatin Versus Concurrent Accelerated Radiation, Cisplatin, and Cetuximab ( C225) [ Followed By Surgery for Selected Patients ] for Stage Iii and Iv Head and Neck Carcinomas. NRG Oncology, USA. 2009.

4. Giaddui T, Chen W, Yu J, et al. Establishing the feasibility of the dosimetric compliance criteria of RTOG 1308:phase III randomized trial comparing overall survival after photon versus proton radiochemotherapy for inoperable stage II-IIIB NSCLC. Radiat Oncol. 2016;11(66).

5. A Li, T Giaddui, W Chen, et al. An Analysis of Rapid Review Cases That Failed to Meet the RTOG 1005 Contouring and Dose-Volume Criteria. Int J Radiat Oncol. 2013;87(2):S228

6. Pinnacle3 release document 9.10 (2014) Radiation Oncology.

7. MIM Vista, Version 6.5. MIM Software INC, Cleveland, OH, 2015.

8. Holt A1 Van Gestel D, Arends MP, Korevaar EW, et al. Multiinstitutional comparison of volumetric modulated arc therapy vs. intensity-modulated radiation therapy for head-and-neck cancer:a planning study. Radiat Oncol. 2013;8(26).

9. Clemente S, Wu B, Sanguineti G, et al. Smart Arc-based volumetric modulated arc therapy for oropharyngeal cancer:A dosimetric comparison with both intensity-modulated radiation therapy and helical tomotherapy. Int J Radiat Oncol Biol Phys. 2011;80(4):1248-1255.

10. Dai X, Zhao Y, Liang Z, et al. Volumetric-modulated arc therapy for oropharyngeal carcinoma:a dosimetric and delivery efficiency comparison with static-field IMRT. Phys Med. 2015;31(1):54-59. 